Article

\title{
Remarks on Distance Based Topological Indices for $\ell$-Apex Trees
}

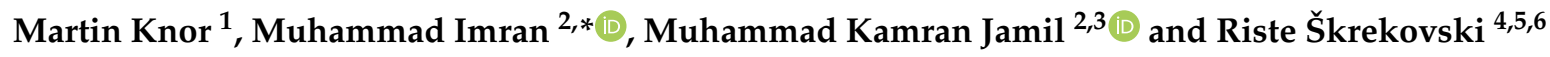 \\ 1 Department of Mathematics, Faculty of Civil Engineering, Slovak University of Technology in Bratislava, \\ 81368 Bratislava, Slovakia; knor@math.sk \\ 2 Department of Mathematical Sciences, Colleges of Sciences, United Arab Emirates University, \\ 15551 Al Ain, UAE; m.imran658@uaeu.ac.ae \\ 3 Department of Mathematics, Riphah Institute of Computing and Applied Sciences, Riphah International \\ University, 54600 Lahore, Pakistan; kamran.jamil@riphah.edu.pk \\ 4 Faculty of Information Studies, 8000 Novo Mesto, Slovenia; Riste.Skrekovski@fmf.uni-lj.si \\ 5 Faculty of Mathematics and Physics, University of Ljubljana, 1000 Ljubljana, Slovenia \\ 6 FAMNIT, University of Primorska, 6000 Koper, Slovenia. \\ * Correspondence: m.imran658@uaeu.ac.ae; Tel.: +971-3-713-6389
}

Received: 7 April 2020; Accepted: 6 May 2020; Published: 12 May 2020

check for updates

\begin{abstract}
A graph $G$ is called an $\ell$-apex tree if there exist a vertex subset $A \subset V(G)$ with cardinality $\ell$ such that $G-A$ is a tree and there is no other subset of smaller cardinality with this property. In the paper, we investigate extremal values of several monotonic distance-based topological indices for this class of graphs, namely generalized Wiener index, and consequently for the Wiener index and the Harary index, and also for some newer indices as connective eccentricity index, generalized degree distance, and others. For the one extreme value we obtain that the extremal graph is a join of a tree and a clique. Regarding the other extreme value, which turns out to be a harder problem, we obtain results for $\ell=1$ and pose some open questions for higher $\ell$. Symmetry has always played an important role in Graph Theory, in recent years, this role has increased significantly in several branches of this field, including topological indices of graphs.
\end{abstract}

Keywords: apex trees; distances in apex trees; generalized Wiener index; extremal graphs

\section{Introduction}

All graphs considered in this paper are simple, finite, connected and undirected. The distance between two vertices $u$ and $v$ is the length of the shortest path between them, it is denoted as $\operatorname{dist}_{G}(u, v)$. If two vertices $u, v$ are adjacent, we write $u \sim v$. The maximum distance from $v \in V(G)$ to any other vertex in $G$ is called the eccentricity, $\varepsilon(v)$, of $v$. For a vertex $v \in V(G), G-v$ is a subgraph obtained by removing the vertex $v$ and all the edges adjacent to $v$. Similarly for any edge $e \in E(G), G-e$ is a subgraph obtained by removing the edge $e$ from $G$. For a subset $U \subset V(G), G-U$ is a subgraph achieved by removing all the vertices in $U$ and all the edges adjacent to any vertex of $U$.

A graph is said to be an apex graph if it contains a vertex whose removal yields a planar graph. In topological graph theory, apex graphs play a vital role [1]. Alkanes play in an important role in chemistry, pharmaceutics and related fields. The apex graph produces trees (alkanes) after removing certain vertices. A graph $G$ is called an $\ell$-apex tree if there exists a vertex subset $A \subset V(G)$ with cardinality $l$ such that $G-A$ is a tree and there is no other subset of smaller cardinality with this property. Elements of the set $A$ are called apex vertices, and $A$ is called an $\ell$-apex vertex set. We denote the set of all $\ell$-apex trees of order $n$ by $\mathcal{A}(n, \ell)$. The elements of the set $\mathcal{A}(n, 1)$ are called just apex trees. Note that a tree is always an 0-apex tree. 
When vertices represent the atoms and the edge between them are the bonds, then the graph $G$ is called a molecular graph. In 1947, Wiener [2] proposed the first topological index, $W(G)$, nowdays called Wiener index,

$$
W(G)=\sum_{\{u, v\} \subseteq V(G)} \operatorname{dist}_{G}(u, v) .
$$

In [2-4], Wiener showed that there exists correlations between the Wiener index of the molecular graph of an organic compound and different physical and chemical properties of the molecular compound. Later were introduced many variatoins, like hyper-Wiener index

$$
\mathrm{WW}(G)=\sum_{\{u, v\} \subseteq V(G)}\left(\operatorname{dist}(u, v)+\operatorname{dist}^{2}(u, v)\right),
$$

and the generalized Wiener index

$$
W^{\lambda}(G)=\sum_{\{u, v\} \subseteq V(G)} \operatorname{dist}^{\lambda}(u, v) .
$$

Notice that for $\lambda=-1$ and 1, we obtain the Wiener index and the Harary index, respectively. For further studies on the Wiener index and its variations, we refer [5-13] and references there in. In the paper we consider also some other newer indices as eccentric connectivity index, generalized degree distance, and others. We define them in the sequel.

In recent years, many papers on $\ell$-apex trees have published. Here we mention only few of them. In [14], authors found the extremal $\ell$-apex trees with respect to matching energy. Xu et al. [15] obtained the sharp bounds on weighted Harary indices for apex trees and $\ell$-apex trees. Extremal first and second Zagreb indices of apex trees have investigated in [16] and they also characterized the extremal $\ell$-apex trees.

\section{One Extremum}

Let $I$ be a graph invariant which is strictly monotonic with respect to adding edges. That is, either for every graph $G$ and every $e \notin E(G)$ we have $I(G+e)<I(G)$, in which case we say that $I$ is decreasing; or for every graph $G$ and every $e \notin E(G)$, we have $I(G+e)>I(G)$, in which case we say that $I$ is increasing.

Let $G$ be an $\ell$-apex tree. Denote by $A$ an $\ell$-apex set of vertices of $G$, add an edge connecting two vertices of $A$ and denote by $G^{\prime}$ the resulting graph. Obviously, $G^{\prime}-A$ is a tree. However, it can happpen that $G^{\prime}$ is a $\kappa$-apex tree for $\kappa<\ell$. For example, take the graph $G$ from Figure 1 consisting of two triangles sharing a vertex. Notice that $G$ has five vertices and it is a 2-apex tree. Then, $A=\left\{u_{2}, u_{3}\right\}$ is a 2-apex set of $G$, but $G+u_{2} u_{3}$ is just a 1-apex tree. Hence to obtain extremal graphs, one cannot apply the monotonicity of $I$ in a random way. Nevertheless, the following holds.

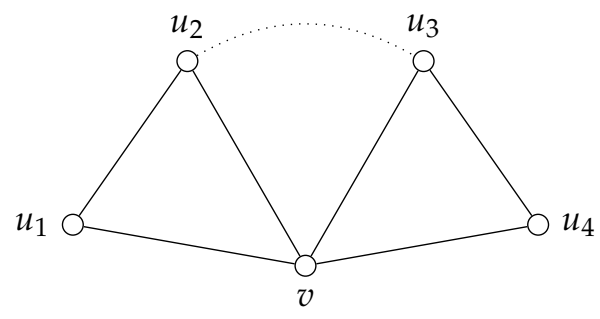

Figure 1. A 2-apex graph that turns into a 1-apex graph by introducing an edge.

Theorem 1. Let $I$ be a strictly monotonic invariant and let $G$ be an $\ell$-apex tree on $n$ vertices, where $\ell \geq 1$ and $n \geq \ell+2$, such that

- $\quad$ G has the minimum possible value of I if I is decreasing; 
- G has the maximum possible value of I if I is increasing.

Then, $G$ is the join $K_{\ell}+T$, where $T$ is a tree on $n-\ell$ vertices.

Proof. We present proof for the case when $I$ is decreasing, the proof for the case when $I$ is increasing is generally the same.

So let $G$ be an $\ell$-apex tree of order $n$ with the minimum value of $I$, and let $A$ be an $\ell$ apex set of vertices of $G$. Then $G-A$ is a tree with $n-\ell$ vertices. Denote this tree by $T$.

Consider all pairs of vertices $u, v$ such that $u \in A$ and $v \neq u$. Some such pairs are edges of $G$, but some are not. Denote by $G^{\prime}$ a graph obtained from $G$ after adding all such pairs $u v$, where $u v \notin E(G)$. Since $I$ is decreasing, we have $I\left(G^{\prime}\right) \leq I(G)$ with equality if and only if $G^{\prime}=G$. It remains to prove that $G^{\prime}$ is $\ell$-apex tree.

Since $G^{\prime}-A$ is $T$, the graph $G^{\prime}$ is a $k$-apex tree for $k \leq \ell$. Let $B$ be a set of vertices of $G^{\prime}$ which has less than $\ell$ vertices. Then $G^{\prime}-B$ has at least 3 vertices. If $B \subset A$ then by the construction of $G^{\prime}$ we have $G^{\prime}-B$ is the join $T+(A-B)$, and since $T$ is a tree on at least 2 vertices, $G^{\prime}-B$ is not a tree. On the other hand if $B \nsubseteq \subset A$ then there are at least two vertices in $A-B$. Let $x, y \in A-B$. Then both $x$ and $y$ are connected to all vertices of $G^{\prime}-B$, and so $G^{\prime}-B$ is not a tree. Thus, $G^{\prime}$ is $\ell$-apex tree.

In what follows, we will apply the above result to several topological indices. We start with generalized Wiener index and several derived indices, afterwards we consider connective eccentricity index, and finally we deal with generalized degree distance.

\subsection{Generalized Wiener Index}

Observe that in $T+K_{\ell}$ all pairs of vertices are at distance 1 with the exception of pairs of non-adjacent vertices of $T$, which are at distance 2. Since there are $\left(\begin{array}{c}n-\ell \\ 2\end{array}\right)-(n-\ell-1)$ such pairs of vertices regardless of the shape of $T$, for invariants depending only on $\operatorname{dist}(u, v)$ the tree $T$ can be arbitrary. Hence, for the generalized Wiener index we have the following corollary.

Corollary 1. Let $G$ be an $\ell$-apex tree on $n$ vertices, where $\ell \geq 1$ and $n \geq \ell+2$, and let $\lambda \neq 0$. Then, the following two claims hold:

- If $\lambda>0$ then $W^{\lambda}(G)$ has the minimum value if and only if $G=K_{\ell}+T$, where $T$ is any tree on $n-\ell$ vertices;

- If $\lambda<0$ then $W^{\lambda}(G)$ has the maximum value if and only if $G=K_{\ell}+T$, where $T$ is any tree on $n-\ell$ vertices.

Moreover, in the extremal case

$$
W^{\lambda}(G)=\left(n^{2}-2 n \ell-3 n+\ell^{2}+3 \ell+2\right) 2^{\lambda-1}+\left(2 n \ell+2 n-\ell^{2}-3 \ell-2\right) 2^{-1} .
$$

Proof. Since $W^{\lambda}$ is decreasing if $\lambda>0$ and increasing if $\lambda<0$, the structural part is a direct consequence of Theorem 1 . Since there are $\left(\begin{array}{c}n-\ell \\ 2\end{array}\right)-(n-\ell-1)$ pairs of vertices of $G$ which distance is 2 while all other $\left(\begin{array}{l}n \\ 2\end{array}\right)-\left(\begin{array}{c}n-\ell \\ 2\end{array}\right)+(n-\ell-1)$ pairs are adjacent, we get the value of $W^{\lambda}(G)$.

The above corollary has consequences on some derived indices.

\subsubsection{Wiener Index.}

Observe that for $\lambda=1$ the invariant $W^{\lambda}$ is the Wiener index, and by Corollary 1 the extremal value is

$$
W(G)=\left(2 n^{2}-2 n \ell-4 n+\ell^{2}+3 \ell+2\right) 2^{-1} .
$$


2.1.2. Harary Index.

On the other hand for $\lambda=-1$ the invariant $W^{\lambda}$ is the Harary index, and by Corollary 1 the extremal value is

$$
H(G)=\left(n^{2}+2 n \ell+n-\ell^{2}-3 \ell-2\right) 2^{-2} .
$$

\subsubsection{Hyper-Wiener Index.}

Summing up $W^{1}$ and $W^{2}$ gives us the hyper-Wiener index $W W$, and again by Corollary 1 regarding the minimum value, the extremal graph is $T+K_{\ell}$ and the extremal value can be again similarly derived as in the previous two indicies

$$
\mathrm{WW}(G)=2 l^{2}-4 \ln +3 n^{2}+6 l-7 n+4 .
$$

\subsection{Connective Eccentricity Index}

Regarding Theorem 1, there are strictly monotonic invariants for which $T$ cannot be arbitrary. One such is connective eccentricity index, defined as

$$
\xi^{c e}(G)=\sum_{v \in V(G)} \frac{\operatorname{deg}(v)}{\varepsilon(v)} .
$$

Mathematical properties and applications of the connective eccentricity index can be found in [17-21], while the authors in [22] discussed the extramal total eccentricity of $l$-apex trees. For this index, we have the following.

Corollary 2. Let $G$ be an $\ell$-apex tree on $n$ vertices, where $\ell \geq 1$ and $n \geq \ell+2$. Then $\xi^{c e}(G)$ has the maximum value if and only if $G=K_{\ell}+T$, where $T$ is the star on $n-\ell$ vertices. Moreover, in the extremal case

$$
\xi^{c e}(G)=\frac{1}{2}(\ell+1)(3 n-\ell-3) .
$$

Proof. Since $\xi^{c e}$ is increasing, the extremal graph is $T+K_{\ell}$ by Theorem 1 . Thus,

$$
\xi^{c e}(G)=\sum_{v \in V(T)} \frac{\operatorname{deg}(v)}{e(v)}+\sum_{v \in V\left(K_{\ell}\right)} \frac{\operatorname{deg}(v)}{e(v)} .
$$

Now the contribution of every $v \in V\left(K_{\ell}\right)$ is $n-1$ since $e(v)=1$ and $\operatorname{deg}(v)=n-1$ in this case. If $v \in V(T)$ then its contribution is $n-1$ if $v$ is connected to all vertices of $T-v$, and $\operatorname{deg}(v) / 2$ otherwise. Notice that the former case happens only if $T$ is a star and $v$ is its center. Since

$$
\begin{aligned}
\sum_{v \in V(T)} \frac{\operatorname{deg}_{G}(v)}{2} & =\frac{1}{2}\left((n-\ell) \ell+\sum_{v \in V(T)} \operatorname{deg}_{T}(v)\right) \\
& =\frac{1}{2}((n-\ell) \ell+2(n-\ell-1)),
\end{aligned}
$$

which is a constant not depending on $T$, for any tree $T$ on $n-\ell$ vertices $\xi^{c \mathcal{e}}(G)$ is a constant with a unique exception when $T$ is the star, for which $\xi^{c e}(G)$ is bigger. Hence, the maximum value of $\xi^{c e}(G)$ is attained when $G=T+K_{\ell}$ when $T$ is the star.

The extremal value of $\xi^{c e}(G)$ could be easily calculated using the fact that $n-\ell-1$ endvertices of $T$ contribute by $(\ell+1) / 2$, while the other $\ell+1$ vertices of $G$ contribute by $n-1$. 


\subsection{Generalized Degree Distance Index}

Analogous situation occures for generalized degree distance

$$
\left.H_{\lambda}(G)=\sum_{u \neq v}(\operatorname{deg}(u)+\operatorname{deg}(v)) \operatorname{dist}^{\lambda}(u, v)\right)
$$

if $\lambda<0$. We have the following result.

Corollary 3. Let $G$ be an $\ell$-apex tree on $n$ vertices, where $\ell \geq 1$ and $n \geq \ell+2$, and let $\lambda<0$. Then $H_{\lambda}(G)$ has the maximum value if and only if $G=K_{\ell}+T$, where $T$ is the star on $n-\ell$ vertices. Moreover, in the extremal case

$$
H_{\lambda}(G)=(\ell+1)\left((n-\ell-1)(n-\ell-2) 2^{\lambda}+n^{2}+n \ell-n-\ell^{2}-2 \ell\right) .
$$

Proof. Since $H_{\lambda}$ is increasing if $\lambda<0$, the extremal graph is $T+K_{\ell}$ by Theorem 1 . Denote $D(u, v)=$ $(\operatorname{deg}(u)+\operatorname{deg}(v)) \operatorname{dist}^{\lambda}(u, v)$. Then

$$
H_{\lambda}(G)=\sum_{\substack{u, v \in V(T) \\ u \neq v}} D(u, v)+\sum_{\substack{u \in V(T) \\ v \in V\left(K_{\ell}\right)}} D(u, v)+\sum_{\substack{u, v \in V\left(K_{\ell}\right) \\ u \neq v}} D(u, v) .
$$

If $u, v \in V\left(K_{\ell}\right)$ then $D(u, v)=2(n-1)$, so the third sum of $(1)$ is $2\left(\begin{array}{l}\ell \\ 2\end{array}\right)(n-1)$ which is a constant depending on $n$ and $\ell$, but not on the structure of $T$. If $u \in V(T)$ and $v \in V\left(K_{\ell}\right)$ then $D(u, v)=$ $(\operatorname{deg}(u)+n-1)$. For fixed $v$, the sum of $D(u, v)$ in the second sum sums to $2(n-\ell-1)+(n-\ell) \ell+$ $(n-\ell)(n-1)$. Hence, also the second sum in (1) is a constant depending on $n$ and $\ell$, but not on the structure of $T$.

Now consider $u, v \in V(T)$. Then

$$
D(u, v)=\left(\operatorname{deg}_{G}(u)+\operatorname{deg}_{G}(v)\right) \operatorname{dist}^{\lambda}(u, v)=\left(\operatorname{deg}_{T}(u)+\operatorname{deg}_{T}(v)+2 \ell\right) \operatorname{dist}^{\lambda}(u, v) .
$$

For $u, v \in V(T)$ exactly $n-\ell-1$ distances are 1 while the others are 2 . Hence, for $u, v \in V(T)$ the sum $\sum 2 \ell \operatorname{dist}^{\lambda}(u, v)$ is a constant, and so it suffices to consider

$$
\sum_{\substack{u, v \in V(T) \\ u \neq v}}\left(\operatorname{deg}_{T}(u)+\operatorname{deg}_{T}(v)\right) \operatorname{dist}^{\lambda}(u, v) .
$$

Observe that running through all distinct $u, v \in V(T)$ also $\sum(\operatorname{deg}(u)+\operatorname{deg}(v))$ is a constant since it equals $(n-\ell-1) \sum_{v \in V(T)} \operatorname{deg}_{T}(v)=2(n-\ell-1)^{2}$. So, we can rewrite (2) in the following way:

$$
\begin{aligned}
= & \sum_{\substack{u, v \in V(T) \\
u \sim v}}\left(\operatorname{deg}_{T}(u)+\operatorname{deg}_{T}(v)\right) 1^{\lambda}+\sum_{\substack{u, v \in V(T) \\
u \neq v, u \neq v}}\left(\operatorname{deg}_{T}(u)+\operatorname{deg}_{T}(v)\right) 2^{\lambda} \\
= & \sum_{\substack{u, v \in V(T) \\
u \sim v}}\left(\operatorname{deg}_{T}(u)+\operatorname{deg}_{T}(v)\right) 1^{\lambda}+\sum_{\substack{u, v \in V(T) \\
u \neq v}}\left(\operatorname{deg}_{T}(u)+\operatorname{deg}_{T}(v)\right) 2^{\lambda} \\
& -\sum_{\substack{u, v \in V(T) \\
u \sim v}}\left(\operatorname{deg}_{T}(u)+\operatorname{deg}_{T}(v)\right) 2^{\lambda} \\
= & \sum_{\substack{u, v \in V(T) \\
u \sim v}}\left(\operatorname{deg}_{T}(u)+\operatorname{deg}_{T}(v)\right)\left(1-2^{\lambda}\right)+\sum_{\substack{u, v \in V(T) \\
u \neq v}}\left(\operatorname{deg}_{T}(u)+\operatorname{deg}_{T}(v)\right) 2^{\lambda} \\
= & \left(1-2^{\lambda}\right) \sum_{\substack{u, v \in V(T) \\
u \sim v}}\left(\operatorname{deg}_{T}(u)+\operatorname{deg}_{T}(v)\right)+(n-\ell-1)^{2} 2^{\lambda+1} .
\end{aligned}
$$


The second sum in the above expression turns to be a constant depending only of $n$ and $\ell$, so the maximum value of $H_{\lambda}$ is achived when we maximize

$$
\sum_{\substack{u, v \in V(T) \\ u \sim v}}\left(\operatorname{deg}_{T}(u)+\operatorname{deg}_{T}(v)\right),
$$

and it is obtained when for all adjacent pairs the sum of degrees $\operatorname{deg}(u)+\operatorname{deg}(v)$ is maximum possible. Since $u$ and $v$ are adjacent vertices in a tree on $n-\ell$ vertices, $\operatorname{deg}_{T}(u)+\operatorname{deg}_{T}(v) \leq n-\ell$. Moreover, every pair of adjacent vertices in $T$ satisfies $\operatorname{deg}_{T}(u)+\operatorname{deg}_{T}(v)=n-\ell$ if and only if every endvertex of $T$ has eccentricity at most 2 in $T$. Therefore the extremal graph is $T+K_{\ell}$ where $T$ is the star on $n-\ell$ vertices.

For calculating the extremal value of $H_{\lambda}$ observe that $n-\ell-1$ vertices of $G$ have degree $\ell+1$ while the other have degree $n-1$. Thus,

$$
H_{\lambda}(G)=\left(\begin{array}{c}
n-\ell-1 \\
2
\end{array}\right)(2 \ell+2) 2^{\lambda}+(n-\ell-1)(\ell+1)(n+\ell)+\left(\begin{array}{c}
\ell+1 \\
2
\end{array}\right)(2 n-2) .
$$

We state here one consequences of the above corollary.

\subsubsection{Additively Weighted Harary Index.}

Observe that for $\lambda=-1$ the invariant $H_{\lambda}$ is additively weighted Harary index (also known as reciprocal degree distance), and by Corollary 3 the extremal value is

$$
\mathrm{HA}(G)=\frac{1}{2}(\ell+1)\left(3 n^{2}-5 n-\ell^{2}-\ell+2\right) .
$$

\section{Other Extremum}

Now we consider the opposite extremum. Since this case is more complicated, we concentrate on 1-apex trees, i.e. apex trees. Though, it will be interesting to be conisdered the case $\ell \geq 2$ itself.

We have:

Theorem 2. Let I be a strictly monotonic invariant and let $G$ be an apex tree on $n \geq 3$ vertices such that

- G has the maximum possible value of I if I is decreasing;

- $G$ has the minimum possible value of I if I is increasing.

Then $G$ is a unicyclic graph and its unique cycle has a vertex of degree 2 .

Proof. We present proof for the case when $I$ is decreasing, the proof for the case when $I$ is increasing is generally the same.

So let $G$ be a 1-apex tree of order $n$ with the maximum value of $I$, and let $a$ be the apex vertex of $G$. Then $G-a$ is a tree with $n-1$ vertices. Denote this tree by $T$.

Suppose first $\operatorname{deg}(a)=1$ then $G$ is a tree, and hence $G$ is not a 1-apex tree. Therefore $\operatorname{deg}(g) \geq 2$.

Now suppose that $\operatorname{deg}(a) \geq 3$. Then, remove one edge incident with $a$ and denote the resulting graph by $G^{\prime}$. Observe that $G^{\prime}$ consists of a tree $T$ and a vertex $a$ incident to at least two vertices of $T$, so $G^{\prime}$ has a cycle. Since $G^{\prime}-a$ is a tree, $G^{\prime}$ is an 1-apex tree, and $I\left(G^{\prime}\right)>I(G)$, a contradiction.

By the above two paragraphs, we conclude that $\operatorname{deg}(a)=2$ and $a$ lies in a unique cycle of $G$. Thus, the claim follows.

\subsection{Generalized Wiener Index}

For the generalized Wiener index $W^{\lambda}$ we have the following result. But before we state it, we give one definition. The dumbbell graph $D_{c}(a, b)$ is obtained from a path $P_{c}=v_{1} v_{2} \cdots v_{c}$ and disjoint 
$K_{a}$ and $K_{b}$ by connecting $v_{1}$ to a vertex of $K_{a}$ and connecting $v_{c}$ to a vertex of $K_{b}$. Thus the order of so constructed graph is $a+b+c$. Note that without loss of generality, we can always assume that $a, b \neq 2$. For an illustration, see the graph shown in Figure 2. If $a, b \geq 3$ then it is an $(a+b-2)$-apex graph, if $a \geq 3$ and $b=1$, then it is a $(a-1)$-apex graph, and finally if $a=b=1$, it is a 0 -apex graph.

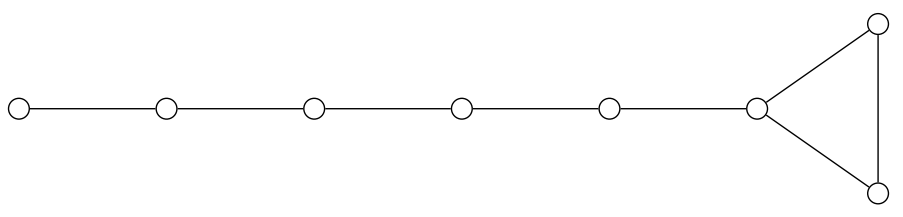

Figure 2. The dumbbell graph $D_{4}(1,3)$.

Corollary 4. Let $G$ be an apex tree on $n \geq 3$ vertices, and let $\lambda \neq 0$. Then the following two claims hold:

- If $\lambda>0$ then $W^{\lambda}(G)$ has the maximum value if and only if $G=D_{n-4}(3,1)$;

- If $\lambda<0$ then $W^{\lambda}(G)$ has the minimum value if and only if $G=D_{n-4}(3,1)$.

Moreover, in the extremal case

$$
W^{\lambda}(G)=1+\sum_{i=1}^{n-2}(n-i) i^{\lambda}
$$

Proof. We present proof for the case $\lambda>0$ when $W^{\lambda}$ is decreasing. The proof for the case $\lambda<0$ when $W^{\lambda}$ is increasing is generally the same.

By Theorem 2, G consists of a tree $T$ and a vertex $a$ of degree two attached to two vertices of $T$. We have

$$
W^{\lambda}(G)=\sum_{u, v \in V(T)} \operatorname{dist}^{\lambda}(u, v)+\sum_{u \in V(T)} \operatorname{dist}^{\lambda}(a, u) .
$$

Denote the vertices of $T$ by $v_{1}, v_{2}, \ldots, v_{n-1}$ so that for every $i, 1 \leq i \leq n-1$, the vertex set $\left\{v_{1}, v_{2}, \ldots, v_{i}\right\}$ induces a connected graph. This connected graph is a tree and we denote it by $T_{i}$. Then $T=T_{n-1}$. For $i \geq 2$, we have

$$
W^{\lambda}\left(T_{i}\right)=W^{\lambda}\left(T_{i-1}\right)+\sum_{j=1}^{i-1} \operatorname{dist}^{\lambda}\left(v_{i}, v_{j}\right)
$$

Since $T_{i}$ is connected, $W^{\lambda}\left(T_{i}\right)-W^{\lambda}\left(T_{i-1}\right)$ is maximum if $\sum_{j=1}^{i-1} \operatorname{dist}^{\lambda}\left(v_{i}, v_{j}\right)=1^{\lambda}+2^{\lambda}+\cdots+$ $(i-1)^{\lambda}$. Since $W^{\lambda}\left(T_{1}\right)=0$ and the maximum for $W^{\lambda}\left(T_{i}\right)-W^{\lambda}\left(T_{i-1}\right)$ is achieved for all $i, 2 \leq i \leq n-1$, only if $T$ is a path, $W^{\lambda}(T)$ is maximum if $T$ is a path on $n-1$ vertices.

Since $a$ is connected to two vertices of $T$, we have

$$
\sum_{u \in V(T)} \operatorname{dist}^{\lambda}(a, u) \leq 1^{\lambda}+1^{\lambda}+2^{\lambda}+3^{\lambda}+\cdots+(n-2)^{\lambda}
$$

and equality is attained only if $T$ is a path on $n-1$ vertices and $a$ is adjacent to one endvertex of $T$ and to its neighbour. Hence, $G$ is $D_{n-4}(3,1)$.

From (3) we get

$$
W^{\lambda}(G)=\sum_{i=1}^{n-2}(n-i-1) i^{\lambda}+1+\sum_{i=1}^{n-2} i^{\lambda}
$$

which gives the formula. 
For $\lambda=1$ the invariant $W^{\lambda}$ is Wiener index, and by Corollary 4 the extremal value is

$$
W(G)=\frac{1}{6}\left(n^{3}-1 n+12\right)
$$

On the other hand for $\lambda=-1$ the invariant $W^{\lambda}$ is Harary index, and by Corollary 1 the extremal value is

$$
H(G)=3-n+n \sum_{i=1}^{n-2} \frac{1}{i} .
$$

It seems to be that $D_{n-4}(3,1)$ is the extremal graph also for $\xi^{\text {ce }}$ if $n \geq 7$. Analogously, $D_{n-4}(3,1)$ seem to be extremal graph also for $H_{\lambda}$ and $H_{* \lambda}$ if $\lambda<0$.

\section{Further Work}

We considered in the paper the extremal values of monotonic distance-based topological indices for the class of $\ell$-apex trees. In particular we have done this for Wiener index, and henceforth for the Wiener index and the Harary index, and also for some newer indices as connective eccentricity index, generalized degree distance, and others. For the one extreme value we obtain that the extremal graph is a join of a tree and a clique. Regarding the other extreme value, which turn to be a harder problem, we obtain results here only for $\ell=1$. Furthermore, it will be a challenge for the readers to consider higher $\ell$.

It seems that the study of topological indices on apex graphs is wide open, and one can take many directions. Just to mention few of them:

- $\quad$ Modified generalized degree distance. This index is defined as

$$
H_{* \lambda}(G)=\sum_{u \neq v} \operatorname{deg}(u) \operatorname{deg}(v) \operatorname{dist}^{\lambda}(u, v)
$$

is increasing if $\lambda<0$. However, it seems to be that if $n-\ell$ is big enough then $H_{* \lambda}(G)$ is maximum if $G=T+K_{\ell}$ where $T$ is the balanced double star.

- Maximum of Wiener index for bigger $\ell$. Let $G$ be an $\ell$-apex tree on $n$ vertices, where $\ell \geq 3$ and $n \geq \ell+1$, such that $G$ has maximum Wiener index. It seems that $G$ is the balanced dumbbell graph. i.e. $G \cong D_{c}(a, b)$, where $a=\lceil\ell / 2\rceil, b=\lfloor\ell / 2\rfloor$, and $c=n-\ell$.

- Minimum of connective eccentricity index for bigger $\ell$. For $n \geq \ell+4$, let $C(n, \ell)$ be the graph obtained from a path $v_{1} v_{2} \cdots v_{n-\ell}$ by connecting $v_{2}$ and $v_{3}$ to every vertex of a stable set of size $n-\ell$. See Figure 3. It is easy to see that $C(n, \ell)$ is an $\ell$-apex graph under assumption that $n-\ell \geq 4$. It seems graphs $C(n, \ell)$ and $D_{n-\ell-1}(\ell+2,1)$ are good candidates for the smallest possible value for the connective eccentricity index.

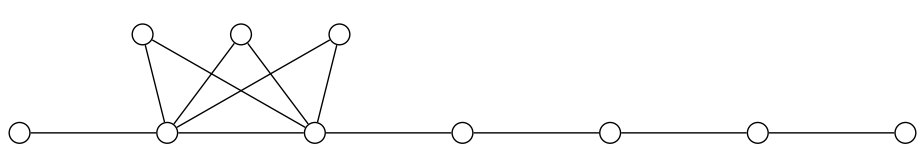

Figure 3. The graph $C(10,3)$.

Author Contributions: All the authors contributed equally. All authors have read and agreed to the published version of the manuscript.

Funding: The research was partially supported by Slovenian research agency ARRS, program no. P1-0383, Slovak research grants APVV-15-0220, APVV-17-0428, VEGA 1/0142/17 and VEGA 1/0238/19. The research was also supported by the UPAR Grants of United Arab Emirates University, Al Ain, UAE via Grant No. G00002590 and G00003271.

Conflicts of Interest: The authors declare no conflict of interest. 


\section{References}

1. Mohar, B. Apex graphs with embeddings of face-width three. Discret. Math. 1997, 176, 203-210. [CrossRef]

2. Wiener, H. Structural determination of paraffin boiling point. J. Am. Chem. Soc. 1947, 69, 17-20. [CrossRef]

3. Wiener, H. Vapor pressure-temperature relationships among the branched paraffin hydroarbons. J. Phys. Chem. 1948, 52, 425-430. [CrossRef]

4. Wiener, H. Correlation of heats of isomerization, and differences in heats of vaporization of isomers, among the paraffin hydroarbons. J. Am. Chem. Soc. 1944, 69, 2636-2638. [CrossRef]

5. Diudea, M.V. Wiener and hyper-Wiener numbers in a single matrix. J. Chem. Inf. Comput. Sci. 1996, 36, 833-836. [CrossRef]

6. Dobrynin, A.A.; Entringer, R.; Gutman, I. Wiener index of trees: Theory and applications. Acta Appl. Math. 2001, 66, 211-249. [CrossRef]

7. Knor, M.; Škrekovski, R. Wiener index of line graphs. In Quantitative Graph Theory: Mathematical Foundations and Applications; Dehmer, M., Emmert-Streib, F., Eds.; CRC Press: Boca Raton, FL, USA, 2014; pp. $279-301$.

8. Knor, M.; Skrekovski, R.; Tepeh, A. Mathematical aspects of Wiener index. ARS Math. Contemp. 2016, 11, 327-352. [CrossRef]

9. Randic, M.; Gou, X.; Oxley, T.; Krishnapriyan, H.; Naylor, L. Wiener matrix invariants J. Chem. Inf. Comput. Sci. 1994, 34, 361-367. [CrossRef]

10. Shang, Y. Bounds of distance Estrada index of graphs. Ars Comb.-Waterloo 2016, 128, 287-294.

11. Shang, Y. Further Results on Distance Estrada Index of Random Graphs. Bull. Malays. Math. Soc. Ser. 2018, 41, 537-544. [CrossRef]

12. Zhou, B.; Gutman, I. Relations between Wiener, hyper-Wiener and Zagreb indices Chem. Phys. Lett. 2004, 394, 93-95.

13. Xu, K.; Liu, M.; Das, K.C.; Gutman, I.; Furtula, B. A Survey on Graphs Extremal with Respect to Distance-Based Topological Indices. MATCH Commun. Math. Comput. Chem. 2014, 17, 461-508.

14. $\mathrm{Xu}, \mathrm{K} . ;$ Zheng, Z.; Das, K.C. Extremal t-apex trees with respect to matching energy. Complexity 2015, 21, 238-247. [CrossRef]

15. Xu, K.; Wang, J.; Das, K.C.; Klavžar, S. Weighted Harary indices of apex trees and k-apex trees. Discret. Appl. Math. 2015, 189, 30-40. [CrossRef]

16. Akhter, N.; Jamil, M.K.; Tomescu, I. Extremal first and second Zagreb indices of apex trees. UPB Sci. Bull. Ser. A 2016, 78, 221-230.

17. De, N.; Nayeem, S.A.; Pal, A. Connective eccentricity index of some thorny graphs. Ann. Pure Appl. Math. 2014, 7, 59-64.

18. Gupta, S.; Singh, M.; Madan, A.K. Connective eccentricity Index: A novel topological descriptor for predicting biological activity. J. Mol. Graph. Model. 2000, 18, 18-25. [CrossRef]

19. Yu, G.; Qu, H.; Tang, L.; Feng, L. On the connective eccentricity index of trees and unicyclic graphs with given diameter. J. Math. Anal. Appl. 2014, 420, 1776-1786. [CrossRef]

20. Xu, K.; Das, K.C.; Liu, H. Some extremal results on the connective eccentricity index of graphs. J. Math. Anal. App. 2016, 433, 803-817. [CrossRef]

21. Yu, G.; Feng, L. On Connective Eccentricity Index of Graphs. MATCH Commun. Math. Comput. Chem. 2013, $69,611-628$.

22. Akhter, N.; Yasin, H.I. Extremal total eccentricity of k-apex trees. Open J. Discret. Appl. Math. 2020, 3, 8-10. [CrossRef]

(C) 2020 by the authors. Licensee MDPI, Basel, Switzerland. This article is an open access article distributed under the terms and conditions of the Creative Commons Attribution (CC BY) license (http:/ / creativecommons.org/licenses/by/4.0/). 